\title{
Endotracheal intubation in the dog
}

In dogs, endotracheal intubation is often necessary for surgery, dental prophylaxis, or other procedures that require gas anesthesia or ventilation. Following a few simple guidelines can help ensure that the procedure is done safely and effectively.

Endotracheal intubation refers to the placement of a tube that extends from the oral cavity into the trachea. The purposes of endotracheal intubation include delivering inhalation anesthetic drugs, ensuring a patent airway in unconscious animals, and administering oxygen to provide ventilatory support. If a study animal becomes unstable during the use of injectable anesthesia, for instance, it may be necessary to provide ventilatory support until the animal is able to breathe again on its own. When routine dental prophylaxis is performed in canine research colonies, intubation is necessary to prevent aspiration during tooth scaling and polishing. Endotracheal intubation is also an important technique for minimizing personnel exposure to waste gas during general anesthesia. Clinical studies involving canine endotracheal intubation include respiratory studies evaluating gaseous exchange and therapeutic drugs, studies of pulmonary obstructive disease, and studies modeling human endotracheal intubation $^{1}$.

\section{PREPARATION}

The appropriate size endotracheal tube is critical to successful intubation. Using a tube that is too large can cause trauma to the larynx and trachea, whereas a tube that is too small will not provide a large enough airway. An internal diameter of $6-8 \mathrm{~mm}$ is appropriate for the average $10-35 \mathrm{lb} \mathrm{dog}^{2}$. The length of the tube should be premeasured against the dog's neck so that the tip of the tube is located midway between the larynx and the thoracic inlet. The tube should be clean and in good condition, and the end of the tube should be lubricated with a small amount of sterile lubricating jelly or water to reduce irritation to the tracheal membranes during intubation.
The endotracheal tube cuff provides a snug seal between the tube and the tracheal wall. The seal prevents escape of anesthetic gases through the trachea and prevents fluid (saliva or blood) from entering the airway. The integrity of the inflatable cuff should be checked prior to the procedure by inserting air into the cuff with a syringe and then sealing the cuff with a stopper or hemostat. A technician should then examine the cuff by listening for leaks or, if there is any doubt about the cuff's integrity, by submerging the cuff in water and looking for bubbles.

\section{RESTRAINT AND POSITIONING}

Endotracheal intubation is performed on animals that are unconscious as a result of trauma, disease, or the administration of sedatives/anesthetic. It is always ideal to have intravenous access (through an intravenous catheter) in any anesthetized patient. Intubation can be performed with the animal in dorsal or lateral recumbency; however, it is most easily performed when an assistant holds the animal in sternal recumbency. The assistant must position the animal so that the head and neck are not twisted to either side. The neck must be extended and the animal's mouth opened wide with one hand holding the upper jaw. Strips of gauze or plastic tubing, placed behind the canine teeth, are particularly useful for holding open the mouth, allowing for greater visualization because the assistant's hands are out the way.

\section{PROCEDURE \\ Intubation}

With the animal in sternal recumbency, the person performing the intubation or the assistant pulls the animal's tongue out of its mouth. A gauze sponge can be used to prevent the tongue from slipping out of one's grasp. Next, locate the larynx with a laryngoscope and depress the epiglottis with the tip of the laryngoscope blade or endotracheal tube to examine the arytenoid cartilages and vocal folds (Fig. 1). Then, pass the lubricated end of the endotracheal tube through the glottis and into the trachea until the tip of the tube is midway between the larynx and thoracic inlet (Fig. 2). If the tube is advanced too far, it can enter the right or left mainstem bronchus, eliminating ventilation of the other lung field.

\section{EOUIPMENT FOR ENDOTRACHEAL TNTUBATION}

Sterile, water-soluble lubricant

Endotracheal tubes of various sizes

Laryngoscope with built-in light source

Gauze squares to grasp the tongue

Roll gauze or plastic tubing to restrain with and tie tube in place

Flexible squeeze bag for mechanical ventilation

5-ml syringe to inflate the endotracheal tube cuff

Stethoscope to assess proper tube placement and monitor the animal

Hemostat to clamp the cuff tube if no valve exists

Additional supplies recommended for anesthetized animals: injectable anesthetic agents, emergency drugs, supplies for i.v. catheter placement, and monitoring equipment 


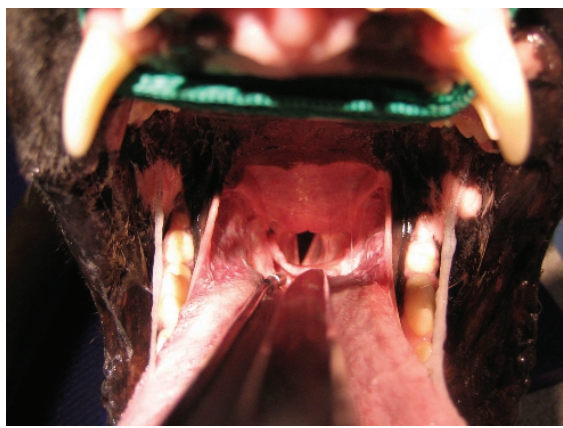

FIGURE 1 | The laryngoscope blade with light source is placed on the tongue and is used to depress the epiglottis and visualize the vocal folds and arytenoid cartilages. The endotracheal tube is placed between the two vocal folds which appear as a ' $V$ ' shape in this image.

Check correct placement of the tube by ausculting both sides of the animal's chest and listening for breathe sounds. Palpating the neck provides further verification of proper tube placement; palpation of two firm tubes in the neck indicates that the esophagus rather than the trachea has been intubated. To secure the tube in place, tie a single half-hitch knot around the tube with a gauze strip (or plastic tubing) and then tie the tube with a quick-release knot around the upper jaw, lower jaw, or behind the ears.

Next, connect the endotracheal tube to an inhalation anesthesia machine, respirator, or flexible squeeze bag. Inflate the cuff of the endotracheal tube with sufficient air to seal the area between the trachea and the tube.

Over-inflation can result in inflammation of the trachea and necrosis. If more than $5 \mathrm{ml}$ of air is necessary to inflate the cuff, this is an indication that a larger diameter tube should have been selected for this animal. When the animal is manually ventilated with a normal tidal volume, no audible air should escape around the cuff, but when the animal is manually ventilated with more than twice its tidal volume, a leak should be heard around the tube, creating a 'safety valve' to protect the lungs from over-inflation.

\section{Monitoring}

Adequate ventilation is indicated by normal-looking, pink-colored mucous membranes and clear lung sounds on auscultation. While the animal is intubated, it is important to make sure the tube does not become kinked as a result of twisting of the neck. The endotracheal tube can become obstructed with secretions, making it necessary to remove these secretions by suctioning through the endotracheal tube. If the plane of anesthesia becomes too light, the reflexes may return, prompting the animal to bite the endotracheal tube and resulting in aspiration of the tube. Appropriate anesthetic monitoring can be accomplished with pulse oximetry, capnography, electrocardiography, Doppler blood pressure monitoring, blood gas analysis, or any combination of these modalities. Body temperature, heart rate, and respirations should always be monitored in the anesthetized animal.

\section{Extubation}

After the primary procedure (which necessitated the anesthesia and intubation) is completed, turn off the flow of inhalant anesthetic but continue administration of oxygen until the animal is ready to be extubated. Loosening the anchoring ties on the tube permits rapid removal of the tube when the animal begins to swallow.

When the swallowing reflex returns, the cuff must be deflated before the tube is removed. Removing the tube with the cuff inflated is traumatic to the trachea and larynx and should be avoided. If there is significant fluid build up around the tube that cannot be effectively suctioned, the cuff may be partially deflated and gently removed to avoid aspiration of fluid proximal to the cuff.

In the semiconscious state following anesthesia, an animal can die as a result of airway obstruction, so careful monitoring for obstruction is necessary until the animal has fully recovered. Position the animal so that the head, neck, and tongue are extended and monitor it until fully conscious. Immediate reintubation may be necessary if concerns arise.

\section{COMPLICATIONS}

Complications associated with endotracheal intubation include the following: trauma to the teeth, mucus membranes of the mouth, soft palate, pharynx, larynx, or tongue; tracheal inflammation or necrosis; subcutaneous emphysema secondary to tracheal trauma; laryngospasm; airway obstruction with secretions; inadequate ventilation due to introduction of the

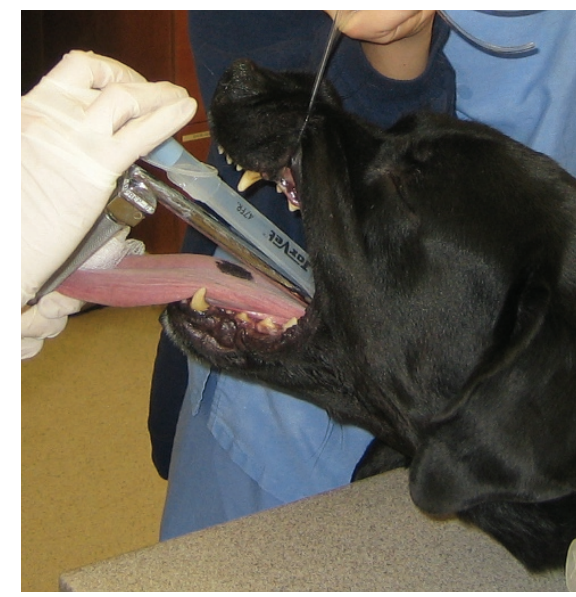

FIGURE 2 | With the dog in sternal recumbency the assistant holds the dog with its neck extended and mouth open. The operator grasps the tongue with a piece of gauze and pulls it out of the mouth as the laryngoscope is used to visualize the glottis and place the endotracheal tube into the trachea.

endotracheal tube into a bronchus; aspiration of endotracheal tube ${ }^{3}$; herniation of the endotracheal tube cuff; kinking of the endotracheal tube during maximum flexion of the atlanto-occipital joint (PVC and wire reinforced endotracheal tubes may be less prone to kinking than red rubber tubes $)^{4}$; vocal cord paralysis ${ }^{5}$; obstructive fibrinous tracheal pseudo-membrane formation ${ }^{6}$; accidental esophageal intubation; and aspiration during extubation ${ }^{7}$.

1. Visaria, R.K. \& Westenskow, D.R. Model-based detection of partially obstructed endotracheal tube. Crit. Care Med. 33(1), 149-154 (2005).

2. Crow, S.E. \& Walshaw, S.0. Manual of Clinical Procedures in the Dog, Cat \& Rabbit 2nd edn. 155-162 (Lippincott-Raven Publishers, Philadelphia, 1997).

3. Bradley, H.W. Removal of bitten off endotracheal tube. Vet. Rec. 102(10), 223-224 (1978).

4. Campoy, L., Hughes, J.M., McAllister, H. \& Bellenger, C.R. Kinking of endotracheal tubes during maxima flexion of the atlanto-occipital joint in dogs. J. Small Anim. Pract. 44(1), 3-7 (2003).

5. Dass, L.L., Sahay, P.N. \& Khan, A.A. Vocal cord paralysis following endotracheal intubation in a bitch. Vet. Rec. 116(8), 218 (1985).

6. Deslee, G. et al. Obstructive fibrinous tracheal pseudomembrane. A potentially fatal complication of tracheal intubation. Am. J. Respir. Crit. Care Med. 162(3 Pt 1), 1169-1171 (2000).

7. Whiffler, K., Andrew, W.K. \& Thomas, R.G. The hazardous cuffed endotracheal tubeaspiration and extubation. S. Afr. Med. J. 61(7), 240-241 (1982). 